\title{
FOURIER MULTIPLIERS ON LIPSCHITZ CURVES
}

\author{
ALAN MCINTOSH AND TAO QIAN
}

\begin{abstract}
We develop the theory of Fourier multipliers acting on $L_{p}(\gamma)$ where $\gamma$ is a Lipschitz curve of the form $\gamma=\{x+i g(x)\}$ with $\|g\|_{\infty}<\infty$ and $\left\|g^{\prime}\right\|_{\infty}<\infty$. The aim is to better understand convolution singular integrals $B$ defined naturally on such curves by
\end{abstract}

$$
B u(z)=\text { p.v. } \int_{\gamma} \varphi(z-\zeta) u(\zeta) d \zeta
$$

for almost all $z \in \gamma$.

\section{INTRODUCTION}

Let $\gamma$ be a Lipschitz curve in the complex plane which has the form $\gamma=$ $\{x+i g(x)\}$ for some function $g$ with Lipschitz constant $N$. Our interest is in the $L_{p}$-boundedness of convolution singular integral operators $B$ defined on $L_{p}(\gamma)$ by

$$
B u(z)=\text { p.v. } \int_{\gamma} \varphi(z-\zeta) u(\zeta) d \zeta
$$

for a suitable function $\varphi$ on $\Gamma=\gamma-\gamma$, and in similar kinds of operators.

Such operators can sometimes be expressed as $B=b\left(D_{\gamma}\right)$ where $D_{\gamma}=$ $d /\left.d z\right|_{\gamma}$ and $b$ is the Fourier transform of $\varphi$. In particular it is known that $B$ is a bounded operator on $L_{p}(\gamma)$ for $1<p<\infty$ if $b$ is a bounded holomorphic function on a double sector $S_{\mu}^{0}=\{z \in \mathbb{C}|| \arg (z) \mid<\mu$ or $|\arg (-z)|<\mu\}$ with $\tan \mu>N$.

When the curve $\gamma$ is contained in a strip $\left\{z|| \mathscr{I}_{m} z \mid \leq M\right\}$ then we can sometimes write $(B u)^{\wedge}=b \hat{u}$ where

$$
\hat{u}(\xi)=\int_{\gamma} e^{-i z \xi} u(z) d z, \quad-\infty<\xi<\infty .
$$

We then call $b$ an $L_{p}(\gamma)$-Fourier multiplier whenever $B$ is a bounded operator on $L_{p}(\gamma)$.

In this paper we shall describe and discuss the spaces $M_{p}(\gamma)$ of $L_{p}(\gamma)$-Fourier multipliers, and give various conditions on $b$ which imply that $b \in M_{p}(\gamma)$. We

Received by the editors April 24, 1989.

1980 Mathematics Subject Classification (1985 Revision). Primary 42B15, 42B20, 47A60.

This research was supported by the Australian government through the Australian Research Council. 
shall discuss the connection between these functions $b$, the operators $b\left(D_{\gamma}\right)$, and convolution operators on $\gamma$.

We have already noted that whenever $b$ extends to a bounded holomorphic function on a double sector $S_{\mu}^{0}$ with $\tan \mu>N$, then $b \in M_{p}(\gamma)$ for $1<p<$ $\infty$. Aside from this, the most interesting condition we give is that whenever $b(\xi) \exp (2 \beta|\xi|) \in M_{p}(\mathbb{R})$ for some $\beta>M$, then $b \in M_{p}(\gamma)$. In particular every bounded measurable function with compact support is an $L_{2}(\gamma)$-Fourier multiplier.

These results have already been announced in [5]. The classical case when $\gamma=\mathbb{R}$ has been treated in a number of places, (e.g., [7]), so we shall not give individual references to the results which we are generalizing.

We would like to thank the many people with whom we have discussed this material, in particular Werner Ricker. This research has been conducted at Macquarie University in Sydney and at the Centre for Mathematical Analysis in Canberra, to both of whom we express our appreciation.

\section{CONVOLUTIONS AND DIFFERENTIATION ON LIPSCHITZ CURVES}

Throughout this paper $\gamma$ denotes the Lipschitz curve consisting of points $x+i g(x) \in \mathbb{C}$, where $x \in \mathbb{R}$ and $g$ is a Lipschitz function which satisfies $\left\|g^{\prime}\right\|_{\infty} \leq N<\infty$. Here $\mathbb{C}$ denotes the complex numbers and $\mathbb{R}$ the reals. We shall use the following spaces of complex valued functions.

If $1 \leq p \leq \infty, L_{p}(\gamma)$ is the space of equivalence classes of functions $u: \gamma \rightarrow$ $\mathbb{C}$ which are measurable with respect to $|d z|$ and for which

$$
\|u\|_{p}=\left\{\int_{\gamma}|u(z)|^{p}|d z|\right\}^{1 / p}<\infty, \quad 1 \leq p<\infty,
$$

or

$$
\|u\|_{\infty}=\operatorname{ess} \sup |u(z)|<\infty .
$$

The space $C_{0}(\gamma)$ is the space of continuous functions $u$ on $\gamma$ which converge to 0 at $\infty$, together with the norm $\|u\|_{\infty}=\sup |u(z)|$.

For $1 \leq p \leq \infty,\left\langle L_{p}(\gamma), L_{p^{\prime}}(\gamma)\right\rangle$ is a dual pair of Banach spaces under $\langle u, v\rangle=\int u(z) v(z) d z$, as is $\left\langle L_{1}(\gamma), C_{0}(\gamma)\right\rangle$, where $p^{\prime}=\left(1-p^{-1}\right)^{-1}$. To be specific, for $1 \leq p \leq \infty$,

$$
\|u\|_{p}=\sup \left\{|\langle u, v\rangle| \mid v \in L_{p^{\prime}}(\gamma),\|v\|_{p^{\prime}}=1\right\},
$$

and

$$
\|u\|_{1}=\sup \left\{|\langle u, v\rangle| \mid v \in C_{0}(\gamma),\|v\|_{\infty}=1\right\} .
$$

Suppose that $\varphi$ is a function defined on a subset of $\mathbb{C}$ which contains $\Gamma=$ $\{z-\zeta \mid z \in \gamma, \zeta \in \gamma\}$, and that $u$ is a measurable function on $\gamma$. Then $\varphi * u$ is defined by $(\varphi * u)(z)=\int_{\gamma} \varphi(z-\zeta) u(\zeta) d \zeta$ for those $z$ for which $\varphi(z-\cdot) u(\cdot) \epsilon$ $L_{1}(\gamma)$.

Theorem 2.1. Let $1 \leq p \leq \infty$. Suppose that $u \in L_{p}(\gamma)$ and that for almost all $z \in \gamma, \varphi(z-\cdot) \in L_{1}(\gamma)$ and $\varphi(\cdot-z) \in L_{1}(\gamma)$. Then

$$
\|\varphi * u\|_{p} \leq \sup _{z \in \gamma}\left\{\int_{\gamma}|\varphi(z-\zeta)||d \zeta|\right\}^{1 / p^{\prime}} \sup _{\zeta \in \gamma}\left\{\int_{\gamma}|\varphi(z-\zeta) \| d z|\right\}^{1 / p}\|u\|_{p}
$$

where $p^{\prime}=\left(1-p^{-1}\right)^{-1}$. 
Proof. First note that $\varphi(z-\cdot) u(\cdot)$ is measurable for almost all $z \in \gamma$. Then, if $1<p<\infty$,

$$
\begin{aligned}
\|\varphi * u\|_{p} & \leq\left\{\int_{\gamma}\left\{\int_{\gamma}|\varphi(z-\zeta) u(\zeta)||d \zeta|\right\}^{p}|d z|\right\}^{1 / p} \\
& =\left\{\int_{\gamma}\left(\int_{\gamma}|\varphi(z-\zeta)|^{1 / p^{\prime}}|\varphi(z-\zeta)|^{1 / p}|u(\zeta)||d \zeta|\right)^{p}|d z|\right\}^{1 / p} \\
& \leq\left\{\int_{\gamma}\left(\int_{\gamma}|\varphi(z-\zeta) \| d \zeta|\right)^{p / p^{\prime}}\left(\int_{\gamma}|\varphi(z-\zeta)||u(\zeta)|^{p}|d \zeta|\right)|d z|\right\}^{1 / p} \\
& \leq \sup _{z \in \gamma}\left(\int_{\gamma}|\varphi(z-\zeta) \| d \zeta|\right)^{1 / p^{\prime}}\left\{\int_{\gamma} \int_{\gamma}|\varphi(z-\zeta)\|d z\| u(\zeta)|^{p}|d \zeta|\right\}^{1 / p} \\
& \leq \sup _{z \in \gamma}\left(\int_{\gamma}|\varphi(z-\zeta) \| d \zeta|\right)^{1 / p^{\prime}} \sup _{\zeta \in \gamma}\left(\int_{\gamma}|\varphi(z-\zeta) \| d z|\right)\|u\|_{p} .
\end{aligned}
$$

The cases $p=1$ and $p=\infty$ have similar proofs.

This theorem will now be used to obtain bounds on the operators $R_{\lambda}$ defined by

$$
R_{\lambda} u=\varphi_{\lambda} * u
$$

where, when $\mathscr{I}_{m} \lambda>0$,

$$
\varphi_{\lambda}(z)= \begin{cases}i e^{i \lambda z}, & \mathscr{R} e z>0 \\ 0, & \mathscr{R} e z \leq 0\end{cases}
$$

and, when $\mathscr{I}_{m} \lambda<0$,

$$
\varphi_{\lambda}(z)= \begin{cases}0, & \mathscr{R} e z \geq 0 \\ -i e^{i \lambda z}, & \mathscr{R} e z<0 .\end{cases}
$$

Let $\omega=\tan ^{-1} N$, and $S_{\omega}=\{z \in \mathbb{C}|| \arg z \mid \leq \omega$ or $|\arg (-z)| \leq \omega\} \cup\{0\}$.

Theorem 2.2. (i) If $\lambda \notin S_{\omega}$, then $R_{\lambda}$ is a bounded operator on $L_{p}(\gamma), 1 \leq p \leq$ $\infty$, and on $C_{0}(\gamma)$, and $\left\|R_{\lambda}\right\| \leq\left\{\operatorname{dist}\left(\lambda, S_{\omega}\right)\right\}^{-1} \sqrt{1+N^{2}}$ in each case.

(ii) If $\|g\|_{\infty} \leq M<\infty$ and $\mathscr{I} m \lambda \neq 0$, then $R_{\lambda}$ is a bounded operator on $L_{p}(\gamma), 1 \leq p \leq \infty$, and on $C_{0}(\gamma)$, and $\left\|R_{\lambda}\right\| \leq\left|\mathscr{S}_{m} \lambda\right|^{-1} \exp (2 M|\mathscr{R e} \lambda|) \sqrt{1+N^{2}}$ in each case.

(iii) Under the assumptions of either (i) or (ii) above, $\left\langle R_{\lambda} u, v\right\rangle=\left\langle u,-R_{-\lambda} v\right\rangle$ for $u \in L_{p}(\gamma), v \in L_{p^{\prime}}(\gamma), 1 \leq p \leq \infty$ (and hence for $u \in L_{1}(\gamma), v \in C_{0}(\gamma)$ ). Proof. The proof is a direct application of Theorem 2.1. For example, if $\mathscr{I}_{m} \lambda>N|\mathscr{R e} \lambda|$ and $z \in \gamma$, then

$$
\begin{aligned}
\int_{\gamma}\left|\varphi_{\lambda}(z-\zeta)\right||d \zeta| & =\int_{\gamma_{z}^{-}}\left|e^{i \lambda(z-\zeta)}\right||d \zeta| \\
& \leq\left\{\operatorname{dist}\left(\lambda, S_{\omega}\right)\right\}^{-1} \sqrt{1+N^{2}} .
\end{aligned}
$$

By $\gamma_{z}^{-}$and $\gamma_{z}^{+}$we denote the curves

$$
\gamma_{z}^{-}=\{\zeta \in \gamma \mid \mathscr{R} e \zeta \leq \mathscr{R e} z\} \quad \text { and } \gamma_{z}^{+}=\{\zeta \in \gamma \mid \mathscr{R} e \zeta \geq \mathscr{R} e z\}
$$


Let us write down explicitly the definition of $R_{\lambda} u$, valid for $u \in L_{p}(\gamma)$ under condition (i) or (ii) above.

$$
R_{\lambda} u(z)= \begin{cases}i \int_{\gamma_{z}^{-}} e^{i \lambda(z-\zeta)} u(\zeta) d \zeta, & I_{m} \lambda>0, \\ -i \int_{\gamma_{z}^{+}} e^{i \lambda(z-\zeta)} u(\zeta) d \zeta, & \mathscr{I}_{m} \lambda<0\end{cases}
$$

Define the derivative of a Lipschitz function $u$ on $\gamma$ by

$$
u^{\prime}(z)=\left.\frac{d}{d z}\right|_{\gamma} u(z)=\lim _{\substack{h \rightarrow 0 \\ z+h \in \gamma}}\left\{\frac{u(z+h)-u(z)}{h}\right\} \text { a.e. }
$$

and note that

$$
\left.\frac{d}{d z}\right|_{\gamma} u(x+i g(x))=\left(1+i g^{\prime}(x)\right)^{-1} \frac{d}{d x} u(x+i g(x)) .
$$

Next use duality to define $D_{\gamma, p}$ to be the closed linear operator with largest domain $\mathscr{D}\left(D_{\gamma, p}\right)$ in $L_{p}(\gamma), 1 \leq p \leq \infty$, or $\mathscr{D}\left(D_{\gamma}, 0\right)$ in $C_{0}(\gamma)$, which satisfies

$$
\left\langle D_{\gamma, p} u, v\right\rangle=\left\langle u, i v^{\prime}\right\rangle
$$

for all Lipschitz functions $v$ on $\gamma$ with compact support.

The following properties of $D_{\gamma, p}$ can be verified either directly on $\gamma$, or by reduction to the properties of the corresponding operator $D_{p}$ in $L_{p}(\mathbb{R})$ or $C_{0}(\mathbb{R})$.

Theorem 2.3. (i) $D_{\gamma, p} u(x+i g(x))=\left(1+i g^{\prime}(x)\right)^{-1} D_{p} u(x+i g(x))$ and

$$
\begin{aligned}
\mathscr{D}\left(D_{\gamma, p}\right) & \left.=\{u \mid u(\cdot+i g(\cdot))) \in \mathscr{D}\left(D_{p}\right)\right\} \\
& =\left\{\begin{array}{l}
W_{p}^{1}(\gamma), \quad 1 \leq p \leq \infty, \\
\Lambda_{0}(\gamma)=\left\{u \in C_{0}(\gamma) \mid u^{\prime} \in C_{0}(\gamma)\right\}, \quad p=0,
\end{array}\right.
\end{aligned}
$$

which is dense in $L_{p}(\gamma)\left(\right.$ or $\left.C_{0}(\gamma)\right)$ except when $p=\infty$. Moreover, for all $p$, the space of Lipschitz functions on $\gamma$ with compact support is dense in $\mathscr{D}\left(D_{\gamma, p}\right)$ under the norm $\|u\|_{p}+\left\|D_{\gamma, p} u\right\|_{p}$ (or $\left.\|u\|_{\infty}+\left\|D_{\gamma, 0} u\right\|_{\infty}\right)$.

(ii) If $1 \leq p \leq \infty, 1 \leq p^{\prime} \leq \infty, 1 / p+1 / p^{\prime}=1$, then

$$
\left\langle D_{\gamma, p} u, v\right\rangle=-\left\langle u, D_{\gamma, p^{\prime}} v\right\rangle, \quad u \in W_{p}^{1}(\gamma), v \in W_{p^{\prime}}^{1}(\gamma),
$$

and

$$
\left\langle D_{\gamma, 1} u, v\right\rangle=-\left\langle u, D_{\gamma, 0} v\right\rangle, \quad u \in W_{1}^{1}(\gamma), v \in \Lambda_{0}(\gamma) .
$$

Each operator has the largest domain under which the equality holds.

(iii) Suppose $\mathscr{I}_{m} \lambda \neq 0$. If either $\lambda \notin S_{\omega}$ or $\|g\|_{\infty} \leq M$, then

$$
\left\langle-\left(D_{\gamma, p}+\lambda I\right) u, R_{\gamma} v\right\rangle=\langle u, v\rangle
$$

for all $u \in \mathscr{D}\left(D_{\gamma, p}\right)$ and $v$ in the appropriate dual space. Consequently $\lambda$ is not in the spectrum of $D_{\gamma, p},\left(D_{\gamma, p}-\lambda I\right)^{-1}=R_{\lambda}$ and so

$$
\left\|\left(D_{\gamma, p}-\lambda I\right)^{-1}\right\| \leq \begin{cases}\left\{\operatorname{dist}\left(\lambda, S_{\omega}\right)\right\}^{-1} \sqrt{1+N^{2}} & \text { if } \lambda \notin S_{\omega}, \\ \left|\mathscr{I}_{m} \lambda\right|^{-1} \exp \left(2 M\left|\mathscr{R}_{e} \lambda\right|\right) \sqrt{1+N^{2}} & \text { if }\|g\|_{\infty} \leq M,\end{cases}
$$

in $L_{p}(\gamma), 1 \leq p \leq \infty$, or in $C_{0}(\gamma)$. 
We shall write $D_{\gamma}$ in place of $D_{\gamma, p}$ when the space in which it is acting is understood.

\section{Functions of $D_{\gamma}$}

There are numerous ways of defining a functional calculus of an operator. For $D_{\gamma}$, it is easy to define $b\left(D_{\gamma}\right)$ when $b \in \Psi\left(S_{\mu}^{0}\right)$, where $\mu>\omega=\tan ^{-1} N$, and

$$
\Psi\left(S_{\mu}^{0}\right)=\left\{\left.b \in H_{\infty}\left(S_{\mu}^{0}\right)|| b(\zeta)|\leq c| \zeta\right|^{s}\left(1+|\zeta|^{2 s}\right)^{-1} \text { for some } c, s>0\right\} .
$$

Here $S_{\mu}^{0}$ denotes the open set $S_{\mu}^{0}=\{\zeta \in \mathbb{C}|| \arg \zeta \mid<\mu$ or $|\arg (-\zeta)|<\mu\}$ and $H_{\infty}\left(S_{\mu}^{0}\right)$ denotes the space of bounded holomorphic functions defined on $S_{\mu}^{0}$. (See Figure 1.)

For $b \in \Psi\left(S_{\mu}^{0}\right)$, the operator $b\left(D_{\gamma}\right)$ can be defined as an operator on $L_{p}(\gamma)$, $1 \leq p \leq \infty$, or on $C_{0}(\gamma)$, by

$$
b\left(D_{\gamma}\right)=\frac{1}{2 \pi i} \int_{\delta} b(\zeta)\left(D_{\gamma}-\zeta I\right)^{-1} d \zeta
$$

where $\delta$ is a path consisting of four rays as indicated. The integral converges absolutely in the operator norm topology and

$$
\left\|b\left(D_{\gamma}\right)\right\| \leq c \int_{\delta}|\zeta|^{s}\left(1+|\zeta|^{2 s}\right)^{-1}|\zeta|^{-1}|d \zeta|<\infty .
$$

It is straightforward to show that the definition of $b\left(D_{\gamma}\right)$ is independent of the precise rays used, that $\left(b_{1} b\right)\left(D_{\gamma}\right)=b_{1}\left(D_{\gamma}\right) b\left(D_{\gamma}\right)$ when $b_{1}$ is also in $\Psi\left(S_{\mu}^{0}\right)$, and that $\left\langle b\left(D_{\gamma}\right) u, v\right\rangle=\left\langle u, b\left(-D_{\gamma}\right) v\right\rangle$ for each of the dualities introduced previously; cf. [3, 4].

The operator $b\left(D_{y}\right)$ can also be expressed as convolution with a suitable function $\varphi$ defined as follows.

Let $\rho_{\theta}$ denote the ray $\{s \exp (i \theta) \mid 0<s<\infty\}$. For $b \in H_{\infty}\left(S_{\mu}^{0}\right)$ and $z \in S_{\mu}^{0}$, define the holomorphic function $\varphi$ on $S_{\mu}^{0}$ by

$$
\varphi(z)=\frac{1}{2 \pi} \int_{\rho_{\theta}} e^{i z \zeta} b(\zeta) d \zeta-\frac{1}{2 \pi} \int_{\rho_{(\pi+\alpha)}} e^{i z \zeta} b(\zeta) d \zeta
$$

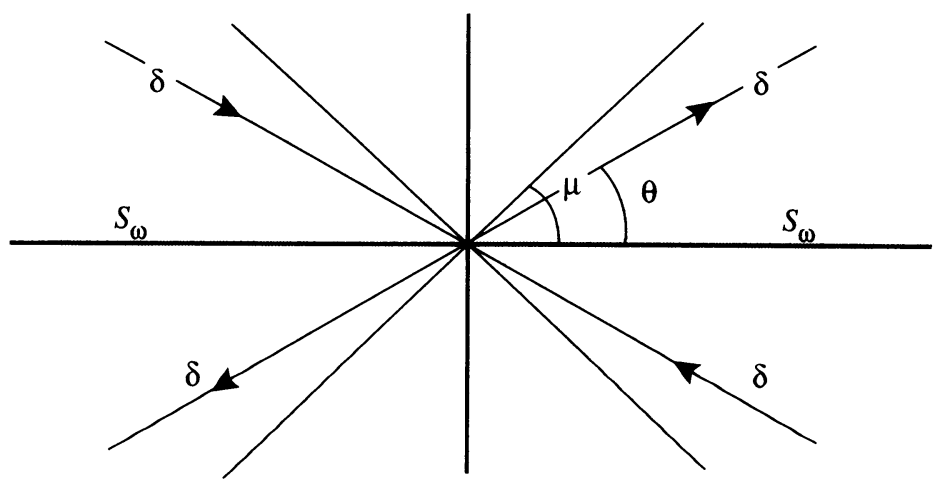

Figure 1 
where $-\mu<-\theta<\arg z<\pi-\theta<\pi+\mu$ and $-\mu<-\alpha<\pi+\arg z<\pi-\alpha<$ $\pi+\mu$. The definition is independent of the precise choice of $\theta$ and $\alpha$. It is not difficult to verify that

$$
|\varphi(z)| \leq\left\{\pi \operatorname{dist}\left(z, \mathbb{C} \sim S_{\mu}^{0}\right)\right\}^{-1}\|b\|_{\infty} .
$$

Under the assumption that $b \in \Psi\left(S_{\mu}^{0}\right)$, that is, $|b(\zeta)| \leq c|\zeta|^{s}\left(1+|\zeta|^{2 s}\right)^{-1}$ where we shall take $0<s<1$, then $\varphi$ satisfies a stronger estimate, and we find that

$$
|\varphi(z)| \leq c|z|^{-1+s}\left(1+|z|^{2 s}\right)^{-1}, \quad z \in S_{\omega} \sim\{0\},
$$

for a different constant $c$. In this case the restriction of $\varphi$ to $\mathbb{R}$ belongs to $L_{1}(\mathbb{R})$, and $b$ is precisely the classical Fourier transform of $\varphi$. That is,

$$
b(\xi)=\int_{\mathbb{R}} e^{-i \xi x} \varphi(x) d x, \quad \xi \in \mathbb{R} .
$$

We leave the verification of this to the reader. A discussion of the more general situation when $b \in H_{\infty}\left(S_{\mu}^{0}\right)$ will be presented in [6].

It is a consequence of the estimate given above for $\varphi$ and of Theorem 2.1 that $\varphi * u \in L_{p}(\gamma)$ whenever $u \in L_{p}(\gamma)$ and that $\varphi * u \in C_{0}(\gamma)$ whenever $u \in C_{0}(\gamma)$. We shall now show that, for $u$ in each of these spaces, $b\left(D_{\gamma}\right) u=\varphi * u$. Indeed, for $u \in L_{p}(\gamma)$ or $C_{0}(\gamma), z \in \gamma$ and $\omega<\theta<\mu$,

$$
\begin{aligned}
(\varphi * u)(z)= & \int_{\gamma} \varphi(z-w) u(w) d w \\
= & \frac{1}{2 \pi}\left[\int_{\gamma_{z}^{-}} \int_{\rho_{\theta}}-\int_{\gamma_{z}^{-}} \int_{\rho_{(\pi-\theta)}}\right. \\
& \left.\quad+\int_{\gamma_{z}^{+}} \int_{\rho_{-\theta}}-\int_{\gamma_{z}^{+}} \int_{\rho_{(\pi+\theta)}}\right] e^{i \zeta(z-w)} b(\zeta) u(w) d \zeta d w \\
= & \frac{1}{2 \pi i} \int_{\delta} b(\zeta)\left(D_{\gamma}-\zeta I\right)^{-1} u(\zeta) d \zeta \\
= & b\left(D_{\gamma}\right) u(z) .
\end{aligned}
$$

Here $\delta$ is the path made up of the rays $-\rho_{-\theta}, \rho_{\theta},-\rho_{(\pi-\theta)}$ and $-\rho_{(\pi+\theta)}$. The estimate proved in Theorem 2.1 has been used to change the order of integration, and then the convolution formula for $\left(D_{\gamma}-\zeta I\right)^{-1}$ given in $\S 2$ has been applied.

\section{The $H_{\infty}$-Functional Calculus of $D_{\gamma}$}

This section is a summary of results initially developed in [2] and in [4]. A full treatment will appear in [6]. Also see [3].

The functional calculus for $D_{\gamma}$ described in the previous section can be extended to all of $H_{\infty}\left(S_{\mu}^{0}\right)$ provided $1<p<\infty$. This means that whenever $b \in H_{\infty}\left(S_{\mu}^{0}\right)$ for some $\mu>\omega$, an operator $b\left(D_{\gamma}\right)$ acting on $L_{p}(\gamma)$ can be defined in a natural way. The following properties hold:

$$
\begin{gathered}
\left\|b\left(D_{\gamma}\right)\right\| \leq c_{\mu}\|b\|_{\infty}, \quad\left(b_{1} b\right)\left(D_{\gamma}\right)=b_{1}\left(D_{\gamma}\right) b\left(D_{\gamma}\right), \\
\left(\alpha_{1} b_{1}+\alpha b\right)\left(D_{\gamma}\right)=\alpha_{1} b_{1}\left(D_{\gamma}\right)+\alpha b\left(D_{\gamma}\right)
\end{gathered}
$$

whenever $b, b_{1} \in H_{\infty}\left(S_{\mu}^{0}\right)$ and $\alpha, \alpha_{1} \in \mathbb{C}$. 
The operator $b\left(D_{\gamma}\right)$ is uniquely determined by the following convergence result. Suppose $\left(b_{n}\right)$ is a sequence of functions in $\Psi\left(S_{\mu}^{0}\right)$ such that

(i) $\left\|b_{n}\right\|_{\infty} \leq$ const $, n=1,2,3, \ldots$;

(ii) for all $0<\delta<\Delta<\infty, b_{n} \rightarrow b$ uniformly on $\left\{\zeta \in S_{\mu}^{0}|\delta \leq| \zeta \mid \leq \Delta\right\}$.

Then $b_{n}\left(D_{\gamma}\right) u \rightarrow b\left(D_{\gamma}\right) u$ for all $u \in L_{p}(\gamma)$.

As well as the function $\varphi$ defined in equation $(*)$ of $\S 3$, a second holomorphic function $\varphi_{1}$ can be defined on $S_{\mu+}^{0}=\left\{z \in S_{\mu}^{0} \mid \mathscr{R} e z>0\right\}$ so that $\varphi$ and $\varphi_{1}$ have the following properties.

(a) $\varphi_{1}^{\prime}(z)=\varphi(z)+\varphi(-z), \quad z \in S_{\mu+}^{0}$;

(b) $\varphi_{1} \in H_{\infty}\left(S_{\nu+}^{0}\right)$ whenever $\omega<\nu<\mu$;

(c) if $u \in L_{p}(\gamma)$, then

$$
b\left(D_{\gamma}\right) u(z)=\lim _{\varepsilon \rightarrow 0}\left\{\int_{\gamma_{z, \varepsilon}} \varphi(z-\zeta) u(\zeta) d \zeta+\varphi_{1}(\varepsilon \mathbf{t}(z)) u(z)\right\}
$$

for almost all $z \in \gamma$, where $\gamma_{z, \varepsilon}=\{\zeta \in \gamma|| z-\zeta \mid \geq \varepsilon\}$ and $\mathbf{t}(z)$ is the unit tangent vector to $\gamma$ at $z$.

This result has a kind of converse which we now state.

Theorem 4.1. Let $\varphi$ and $\varphi_{1}$ be holomorphic functions on $S_{\mu}^{0}$ and $S_{\mu+}^{0}$ respectively, where $\mu>\omega$, and suppose that

(i) $\varphi_{1}^{\prime}(z)=\varphi(z)+\varphi(-z), \quad z \in S_{\mu+}^{0}$,

(ii) $\varphi_{1} \in H_{\infty}\left(S_{\mu+}^{0}\right)$ and $z \varphi(z) \in H_{\infty}\left(S_{\mu}^{0}\right)$.

Let $1<p<\infty$. For $u \in L_{p}(\gamma)$, let

$$
(B u)(z)=\lim _{\varepsilon \rightarrow 0}\left\{\int_{\gamma_{z, \varepsilon}} \varphi(z-\zeta) u(\zeta) d \zeta+\varphi_{1}(\varepsilon \mathbf{t}(z)) u(z)\right\}
$$

where $\mathbf{t}(z)$ and $\gamma_{z, \varepsilon}$ are specified above. Then $(B u)(z)$ is defined for almost all $z \in \gamma$, and $B$ is a bounded linear operator on $L_{p}(\gamma)$. Moreover $B=b\left(D_{\gamma}\right)$ for some $b \in H_{\infty}\left(S_{\nu}^{0}\right)$ and some $\nu>\omega$.

In particular, if $\varphi$ is odd and $\varphi_{1}=0$, then $(B u)(z)=$ p.v. $\int_{\gamma} \varphi(z-\zeta) u(\zeta) d \zeta$.

The best known special case of these results occurs when $b(\zeta)=\operatorname{sgn}(\zeta)$, where $\operatorname{sgn}(\zeta)$ is defined on $S_{\mu}^{0}$ by

$$
\operatorname{sgn}(\zeta)= \begin{cases}1, & \mathscr{R} e \zeta>0, \\ -1, & \mathscr{R} e \zeta<0 .\end{cases}
$$

In this case $\varphi(z)=i(\pi z)^{-1}, \varphi_{1}(z)=0$, and $B$ is the principal-value Cauchy integral $C_{\gamma}$ defined by

$$
C_{\gamma} u(z)=\text { p.v. } \frac{i}{\pi} \int_{\gamma} \frac{1}{z-\zeta} u(\zeta) d \zeta .
$$

The boundedness of $C_{\gamma}$ on $L_{p}(\gamma)$ was first proved in [1].

\section{FOURIER TRANSFORMS ON CURVES IN A STRIP}

We turn now to a more detailed study of the case when the function $g$ defining $\gamma$ satisfies $\|g\|_{\infty} \leq M<\infty$ as well as $\left\|g^{\prime}\right\|_{\infty} \leq N<\infty$. To do this we introduce the following Banach spaces for $-\infty<\beta<\infty$ :

$$
\mathscr{E}_{\beta}=\left\{w:(-\infty, \infty) \rightarrow \mathbb{C} \mid w \text { is Lebesgue measurable and }\|w\|_{\mathscr{E}_{\beta}}<\infty\right\},
$$


and

$$
\mathscr{E}_{\beta}^{2}=\left\{w \in \mathscr{E}_{\beta} \mid w^{\prime}, w^{\prime \prime} \in \mathscr{E}_{\beta}\right\}
$$

where

$$
\|w\|_{\mathscr{E}_{\beta}}=\left\{\int_{-\infty}^{\infty}|w(\xi)|^{2} \exp (2 \beta|\xi|) d \xi\right\}^{1 / 2}
$$

and

$$
\|w\|_{\mathscr{E}_{\beta}^{2}}=\left\{\|w\|_{\mathscr{E}_{\beta}}^{2}+\left\|w^{\prime}\right\|_{\mathscr{E}_{\beta}}^{2}+\left\|w^{\prime \prime}\right\|_{\mathscr{E}_{\beta}}^{2}\right\}^{1 / 2}
$$

We regard $\mathscr{E}_{\beta}^{2}$ as a test space with dual $\left(\mathscr{E}_{\beta}^{2}\right)^{\prime}$ and embed $\mathscr{E}_{-\beta}$ in $\left(\mathscr{E}_{\beta}^{2}\right)^{\prime}$ using $\langle w, v\rangle=\int_{-\infty}^{\infty} w(\xi) v(\xi) d \xi$ when $w \in \mathscr{E}_{-\beta}$ and $v \in \mathscr{E}_{\beta}^{2}$, noting that $|\langle w, v\rangle| \leq$ $\|w\|_{\mathscr{E}_{-\beta}}\|v\|_{\mathscr{E}_{\beta}^{2}}$, and that $\langle w, v\rangle=0$ for all $v \in \mathscr{E}_{\beta}^{2}$ if and only if $w=0$. We remark that if $\alpha<\beta$ then $\mathscr{E}_{\beta} \subset \mathscr{E}_{\alpha}$ and $\mathscr{E}_{\beta}^{2} \subset \mathscr{E}_{\alpha}^{2}$ where the embeddings are continuous and dense, so $\left(\mathscr{E}_{\alpha}^{2}\right)^{\prime} \subset\left(\mathscr{E}_{\beta}^{2}\right)^{\prime}$.

The following definitions of Fourier transform and inverse Fourier transform will be used throughout the paper.

If $u \in L_{1}(\gamma)$ define

$$
\hat{u}(\xi)=\int_{\gamma} e^{i z \xi} u(z) d z .
$$

Then $\hat{u}$ is a continuous function satisfying

$$
|\hat{u}(\xi)| \leq e^{|\xi| M}\|u\|_{1},
$$

so $\hat{u} \in \mathscr{E}_{-\beta}$ for all $\beta>M$, and $\|\hat{u}\|_{\mathscr{E}_{-\beta}} \leq(\beta-M)^{-1 / 2}\|u\|_{1}$.

For $\beta>M$ and $w \in \mathscr{E}_{\beta}$, define the holomorphic function $\check{w}$ on the strip

$$
X_{\beta}=\left\{\zeta \in \mathbb{C}|| \mathscr{I}_{m} \zeta \mid<\beta\right\}
$$

by

$$
\check{w}(\zeta)=\frac{1}{2 \pi} \int_{-\infty}^{\infty} e^{i \zeta \xi} w(\xi) d \xi,
$$

and note (by considering real $\zeta$ ) that if $\check{w}=0$ then $w=0$. Let

$$
\left(\mathscr{E}_{\beta}\right)^{\vee}(\gamma)=\left\{\left.\check{w}\right|_{\gamma} \mid w \in \mathscr{E}_{\beta}\right\} \quad \text { and }\left(\mathscr{E}_{\beta}^{2}\right)^{\vee}(\gamma)=\left\{\left.\check{w}\right|_{\gamma} \mid w \in \mathscr{E}_{\beta}^{2}\right\}
$$

and equip these spaces with the norms $\|\check{w}\|_{\left(\mathscr{E}_{\beta}\right)^{\vee}(\gamma)}=\|w\|_{\mathscr{E}_{\beta}}$ and $\|\check{w}\|_{\left(\mathscr{E}_{\beta}^{2}\right)^{\vee}(\gamma)}=$ $\|w\|_{\mathscr{E}_{\beta}^{2}}$.

Theorem 5.1. (i) A holomorphic function $f$ on $X_{\beta}$ belongs to $\left(\mathscr{E}_{\beta}\right)^{\vee}$ if and only if

$$
\sup _{|y|<\beta} \int|f(x+i y)|^{2} d x<\infty \text {. }
$$

Moreover

$$
\frac{1}{2}\|w\|_{\mathscr{E}_{\beta}} \leq \sqrt{2 \pi} \sup _{|y|<\beta}\left\{\int|\check{w}(x+i y)|^{2} d x\right\}^{1 / 2} \leq\|w\|_{\mathscr{E}_{\beta}} .
$$

(ii) If $w \in \mathscr{E}_{\beta}$ and $\left|\mathscr{I}_{m} z\right| \leq M$, then

$$
|\check{w}(z)| \leq \frac{1}{2 \pi}(\beta-M)^{-1}\|w\|_{\mathscr{E}_{\beta}}
$$


and

$$
\sup _{|y| \leq M}|\check{w}(x+i y)| \rightarrow 0 \text { as }|x| \rightarrow \infty,
$$

so $\left.\check{w}\right|_{\gamma} \in C_{0}(\gamma)$. Hence $\left(\mathscr{E}_{\beta}\right)^{\vee}(\gamma)$ is continuously embedded in $C_{0}(\gamma)$.

(iii) If $w, v \in \mathscr{E}_{\beta}$, then

$$
\int_{\gamma} \check{w}(z) \check{v}(z) d z=\frac{1}{2 \pi} \int_{-\infty}^{\infty} w(\xi) v(-\xi) d \xi .
$$

(iv) A holomorphic function $f$ on $X_{\beta}$ belongs to $\left(\mathscr{E}_{\beta}^{2}\right)^{\vee}$ if and only if

$$
\sup _{|y|<\beta} \int\left|\left(1+x^{2}\right) f(x+i y)\right|^{2} d x<\infty \text {. }
$$

Moreover, there exists $c_{\beta}>0$ such that

$$
\frac{1}{c_{\beta}}\|w\|_{\mathscr{E}_{\beta}^{2}} \leq \sup _{|y|<\beta}\left\{\int\left|\left(1+x^{2}\right) \check{w}(x+i y)\right|^{2} d x\right\}^{1 / 2} \leq c_{\beta}\|w\|_{\mathscr{E}_{\beta}^{2}} .
$$

(v) $\left(\mathscr{C}_{\beta}^{2}\right)^{\vee}(\gamma)$ is continuously embedded in $L_{p}(\gamma)$ for all $p$ such that $1 \leq p \leq$ $\infty$.

Proof. The various estimates for $\check{w}$ are straightforward, the second part of (ii) being deduced from Cauchy's formula on rectangles with vertices $\left(1 \pm \frac{1}{2}\right) \mathscr{R e} z \pm$ $\frac{1}{2} i(M+\beta)$. Because of part (ii), the Cauchy theorem can be used to show that $\int_{\gamma} \check{w}(z) \check{v}(z) d z=\int_{\mathbb{R}} \check{w}(x) \check{v}(x) d x$ so (iii) follows from the usual Parseval's equation. It is also not difficult to see that functions $f$ with the stated properties are of the form $f=\check{w}$ for $w \in \mathscr{E}_{\beta}$ or $\mathscr{E}_{\beta}^{2}$. To prove part (v), first show that if $w \in \mathscr{E}_{\beta}^{2}$, and $|y| \leq M$, then $\left(1+x^{2}\right)|\check{w}(x+i y)| \leq c\|w\|_{\mathscr{E}_{\beta}^{2}}$ for some constant $c$.

We remark that $\bigcup_{\beta>M}\left(\mathscr{E}_{\beta}\right)^{\vee}(\gamma)$ is the space $\mathscr{A}(\gamma)$ used by Coifman and Meyer [2].

In the next theorem we state some density. results and another version of Parseval's formula.

Theorem 5.2. Let $\beta>\alpha>M$, and $1 \leq p<\infty$.

(i) The following inclusions are all dense:

$$
\begin{aligned}
& \left(\mathscr{E}_{\beta}^{2}\right)^{\vee}(\gamma) \subset L_{1} \cap L_{p} \cap\left(\mathscr{E}_{\beta}\right)^{\vee}(\gamma) \subset L_{p} \cap\left(\mathscr{E}_{\beta}\right)^{\vee}(\gamma) \subset\left(\mathscr{E}_{\beta}\right)^{\vee}(\gamma) \\
& \begin{array}{cccc}
\cap & \cap & \cap & \cap \\
\left(\mathscr{E}_{\alpha}^{2}\right)^{\vee}(\gamma) & L_{1} \cap L_{p}(\gamma) & L_{p} \cap\left(\mathscr{E}_{\alpha}\right)^{\vee}(\gamma) & \left(\mathscr{E}_{\alpha}\right)^{\vee}(\gamma)
\end{array} \\
& \cap \cap \cap \\
& L_{p}(\gamma) \quad L_{p}(\gamma)
\end{aligned}
$$

(ii) In the above, $L_{p}(\gamma)$ can be replaced by $C_{0}(\gamma)$.

(iii)

$$
\int_{\gamma} u(z) \check{w}(z) d z=\frac{1}{2 \pi} \int_{-\infty}^{\infty} \hat{u}(\xi) w(-\xi) d \xi
$$

for all $u \in L_{1}(\gamma)$ and $w \in \mathscr{E}_{\beta}$.

(iv) If $u \in L_{1}(\gamma)$ and $\check{u}=0$, then $u=0$.

The horizontal inclusions are dense for the following reason. Let $u \in$ $\left(\mathscr{E}_{\beta}\right)^{\vee}(\gamma)$. For each $\varepsilon>0$, define $u_{\varepsilon}(z)=\left(1+\varepsilon^{2} z^{2}\right)^{-1} u(z)$. By parts (i) 
and (iv) of the proposition above, $u_{\varepsilon} \in\left(\mathscr{E}_{\beta}^{2}\right)^{\vee}(\gamma)$. Also $u_{\varepsilon} \rightarrow u$ in $L_{p}(\gamma)$ and in $C_{0}(\gamma)$. So these inclusions are dense. We have already noted that $\left(\mathscr{E}_{\beta}^{2}\right)^{\vee}(\gamma)$ is dense in $\left(\mathscr{E}_{\alpha}^{2}\right)^{\vee}(\gamma)$, and $\left(\mathscr{E}_{\beta}\right)^{\vee}(\gamma)$ is dense in $\left(\mathscr{E}_{\alpha}\right)^{\vee}(\gamma)$.

The remainder of the proof will be deferred until after we have introduced some approximation techniques.

We see immediately from Theorem 5.1 that the following definition of Fourier transform is consistent with the preceding one for functions $u \in L_{1}(\gamma)$.

If $u \in L_{p}(\gamma)$ for some $p$ such that $1 \leq p \leq \infty$, define $\hat{u} \in\left(\mathscr{E}_{\beta}^{2}\right)^{\prime}$ by $\left\langle\hat{u}, w_{-}\right\rangle=\int_{\gamma} u(z) \check{w}(z) d z$ for all $w \in \mathscr{E}_{\beta}^{2}$, where $w_{-}(\xi)=w(-\xi)$.

Note that this definition is independent of $\beta>M$, and that the mapping $\mathscr{F}: L_{p}(\gamma) \rightarrow\left(\mathscr{E}_{\beta}^{2}\right)^{\prime}$ defined by $\mathscr{F}(u)=\hat{u}$ is continuous and one-one. (We are using the fact that $\left(\mathscr{E}_{\beta}^{2}\right)^{\vee}(\gamma)$ is densely and continuously embedded in $L_{p}(\gamma)$ when $1 \leq p<\infty$ and in $C_{0}(\gamma)$, and that $\left\langle L_{p}(\gamma), L_{p^{\prime}}(\gamma)\right\rangle$ and $\left\langle L_{1}(\gamma), C_{0}(\gamma)\right\rangle$ are dual pairs of Banach spaces.)

Whenever it makes sense, the Fourier transform and the inverse Fourier transform defined above are inverse operations:

Theorem 5.3. Let $u \in L_{p}(\gamma)$ for some $p$ such that $1 \leq p \leq \infty$. Let $w \in \mathscr{E}_{\beta}$ for some $\beta>M$. Then $u=\check{w}$ if and only if $w=\hat{u}$.

The proof of this theorem is also deferred till later in this section.

In the approximation arguments we shall use the maximal function $M_{\gamma} u$ defined for locally integrable functions $u$ on $\gamma$ by

$$
M_{\gamma} u(z)=\sup _{\rho>0} \rho^{-1} \int_{B(z, \rho)}|u(\zeta)||d \zeta|
$$

where $z \in \gamma$ and $B(z, \rho)=\{\zeta \in \gamma|| \zeta-z \mid<\rho\}$. The following propositions can be proved by the usual methods. Recall that $N \geq\left\|g^{\prime}\right\|_{\infty}$.

Proposition. There exist constants $c_{p, N}$ for $1<p \leq \infty$ and $c_{N}$ such that

$$
\left\|M_{\gamma} u\right\|_{p} \leq c_{p, N}\|u\|_{p}, \quad u \in L_{p}(\gamma)
$$

and

$$
\lambda l\left\{z \in \gamma \mid M_{\gamma} u(z)>\lambda\right\} \leq c_{N}\|u\|_{1}, \quad u \in L_{1}(\gamma), \lambda>0 .
$$

Here $l$ denotes the measure introduced by arc-length.

Proposition. Suppose that $u$ is a locally integrable function on $\gamma$ and that $\varphi * u$ and $\psi * u$ are well defined, where $\psi$ is a decreasing function in $L_{1}(0, \infty)$ such that

$$
|\varphi(z)| \leq \psi(|x|), \quad z=x+i y \in \Gamma,
$$

where $\Gamma=\{z-\zeta \mid z, \zeta \in \gamma\}$. Then, for all $z \in \gamma$,

$$
|\varphi * u(z)| \leq c_{N}\|\psi\|_{1} M_{\gamma} u(z) \text {. }
$$

The latter result has a straightforward proof for functions $\psi$ of the form $\psi(\zeta)=\sum_{k} a_{k} \chi_{k}(\zeta)$, where $\chi_{k}$ is the characteristic function of a ball with centre 0 . For general $\psi$, use a sequence of such functions to approach $\psi$ from below. 
The approximation arguments also involve showing that a function $u$ is the limit of a sequence $\varphi_{n} * u$ in an appropriate sense, where $\varphi_{n}(z)=n \varphi(n z)$ and $\varphi$ is a holomorphic function defined on all of $\mathbb{C}$ which satisfies

$$
\int_{-\infty}^{\infty} \varphi(x) d x=1
$$

and

$$
|\varphi(z)| \leq \frac{c}{1+x^{2}}, \quad z=x+i y \in S_{\mu}^{0}
$$

for some constant $c$, where $\tan \mu>N$.

When $N<\pi / 4$ we can take $f(z)=\exp \left(-z^{2}\right)$. For $\pi / 4 \leq N<\pi / 2$, there do exist such functions $\varphi$, as will be shown in the appendix. We shall call a sequence $\left(\varphi_{n}\right)$ constructed as above an identity sequence.

Note the following properties. Let $\psi_{n}(s)=n\left(1+n^{2} s^{2}\right)^{-1}, s>0$. Then

(3) For each $n,\left|\varphi_{n}(z)\right| \leq c \psi_{n}(|x|), z=x+i y \in S_{\mu}^{0}$.

(4) For each $n, \int_{0}^{\infty} \psi_{n}(s) d s=\frac{1}{2} \pi$.

(5) For all $\delta>0, \int_{\delta}^{\infty} \psi_{n}(s) d s \rightarrow 0$ as $n \rightarrow \infty$.

(6) For each $n$ and each $\zeta \in \gamma, \int_{\gamma} \varphi_{n}(z-\zeta) d z=1$.

The following two theorems specify ways in which $\varphi_{n} * u$ converges to $u$.

Theorem 5.4. Let $\left(\varphi_{n}\right)$ be an identity sequence. Then

(i) there exist constants $c_{p, N}$ for $1<p \leq \infty$ such that

$$
\left\|\sup _{n}\left|\varphi_{n} * u\right|\right\|_{p} \leq c_{p, N}\|u\|_{p}, \quad u \in L_{p}(\gamma),
$$

(ii) if $u \in L_{p}(\gamma), 1 \leq p \leq \infty$, then, for almost all $z \in \gamma$,

$$
\lim _{n \rightarrow \infty}\left(\varphi_{n} * u\right)(z)=u(z),
$$

(iii) if $u \in L_{p}(\gamma), 1 \leq p<\infty$, then

$$
\lim _{n \rightarrow \infty}\left\|\left(\varphi_{n} * u\right)-u\right\|_{p}=0
$$

(iv) if $u \in C_{0}(\gamma)$, then

$$
\lim _{n \rightarrow \infty}\left\|\left(\varphi_{n} * u\right)-u\right\|_{\infty}=0
$$

Proof. Part (i) is a consequence of the previous two propositions. Next suppose that $u \in C_{0}(\gamma)$. By property (6),

$$
\begin{aligned}
\left(\varphi_{n} * u\right)(z)-u(z)= & \int_{|\zeta-z|<\delta} \varphi_{n}(z-\zeta)(u(\zeta)-u(z)) d \zeta \\
& +\int_{|\zeta-z| \geq \delta} \varphi_{n}(z-\zeta)(u(\zeta)-u(z)) d \zeta \\
& =\mathrm{I}_{1}+\mathrm{I}_{2} .
\end{aligned}
$$

Let $\varepsilon>0$. Choose $\delta$ small enough that $|u(\zeta)-u(z)|<\varepsilon$ for all $\zeta \in \gamma$ with $|\zeta-z|<\delta$. Therefore, by properties (3) and (4),

$$
\mathrm{I}_{1} \leq \varepsilon \int_{\gamma}\left|\varphi_{n}(z-\zeta)\right||d \zeta| \leq c \varepsilon \pi \sqrt{1+N^{2}}
$$


An application of property (5) now shows that $I_{2} \leq \varepsilon$ for all sufficiently large $n$. Hence

$$
\left|\varphi_{n} * u(z)-u(z)\right| \leq \varepsilon\left(1+c \pi \sqrt{1+N^{2}}\right)
$$

Part (iv) now follows, as does part (ii) in the case when $u \in C_{0}(\gamma)$.

For $u \in L_{p}(\gamma), 1 \leq p<\infty$, and any $\delta>0$, there is a decomposition $u=v+w$ where $v \in C_{0}(\gamma)$ and $\|w\|_{p}<\delta$. Therefore, by the preceding propositions,

$$
\begin{aligned}
l\{z & \left.\in \gamma\left|\varlimsup_{n \rightarrow \infty}\right| \varphi_{n} * u(z)-u(z) \mid>\kappa\right\} \\
& =l\left\{z \in \gamma\left|\varlimsup_{n \rightarrow \infty}\right| \varphi_{n} * w(z)-w(z) \mid>\kappa\right\} \\
& \leq l\left\{z \in \gamma\left|\varlimsup_{n \rightarrow \infty}\right| \varphi_{n} * w(z) \mid>\frac{1}{2} \kappa\right\}+l\left\{z \in \gamma|| w(z) \mid>\frac{1}{2} \kappa\right\} \\
& \leq l\left\{z \in \gamma \mid M_{\gamma} w(z)>\frac{1}{2} \kappa\right\}+l\left\{z \in \gamma|| w(z) \mid>\frac{1}{2} \kappa\right\} \\
& \leq c \kappa^{-p}\|w\|_{p}^{p} \leq c \kappa^{-p} \delta^{p} .
\end{aligned}
$$

Let $\delta \rightarrow 0$ first and then let $\kappa \rightarrow 0$. We conclude that

$$
l\left\{z \in \gamma\left|\varlimsup_{n \rightarrow \infty}\right| \varphi_{n} * u(z)-u(z) \mid>0\right\}=0 .
$$

This identity gives (ii) when $1 \leq p<\infty$. The case $p=\infty$ can be reduced to the case $p=1$ by a localization argument.

(iii) For $u \in L_{p}(\gamma)$, define $U \in L_{p}(\mathbb{R})$ by $U(x)=u(x+i g(x))$. Then

$$
\begin{aligned}
\left\|\left(\varphi_{n} * u\right)-u\right\|_{p} & =\left\|\int_{\gamma} \varphi_{n}(\cdot-\zeta)(u(\zeta)-u(\cdot)) d \zeta\right\|_{L_{p}(y)} \\
& \leq c\left\|\int_{\mathbb{R}} \psi_{n}(|x-y|)|U(x)-U(y)| d y\right\|_{L_{p}(d x)} \\
& =c\left\|\int_{\mathbb{R}} \psi(|s|)\left|U(x)-U\left(x-\frac{s}{n}\right)\right| d s\right\|_{L_{p}(d x)} \\
& \leq c \int_{\mathbb{R}} \psi(|s|)\left\|U(x)-U\left(x-\frac{s}{n}\right)\right\|_{L_{p}(d x)} d s \\
& =c \int_{\mathbb{R}} \psi(|s|) \Delta\left(U, \frac{s}{n}\right) d s
\end{aligned}
$$

where

$$
\Delta\left(U, \frac{s}{n}\right)=\left\|U(x)-U\left(x-\frac{s}{n}\right)\right\|_{L_{p}(d x)} .
$$

Note that $\Delta(U, s / n) \rightarrow 0$ as $n \rightarrow \infty$ and $\Delta(U, s / n) \leq 2\|U\|_{p}$. By the Lebesgue dominated convergence theorem, the last integral tends to zero as $n \rightarrow \infty$.

For the identity sequence $\left(\varphi_{n}\right)$ given by $\varphi_{n}(z)=n \varphi(n z)$ where $\varphi$ satisfies (1) and (2), let $\Phi_{n}=\hat{\varphi}_{n}$ and $\Phi=\hat{\phi}$ (the classical Fourier transforms). Then $\Phi_{n}(\xi)=\Phi\left(n^{-1} \xi\right)$ where $\Phi$ is a continuous function which satisfies $\Phi(0)=1$.

For each $\lambda>0$ there exists $c_{\lambda}$ such that

$$
|\Phi(\xi)| \leq c_{\lambda} e^{-\lambda|\xi|}, \quad-\infty<\xi<\infty
$$


To see this for $\xi>0$ note that

$$
\begin{aligned}
|\Phi(\xi)| & =\left|\int_{-\infty}^{\infty} e^{-i \xi x} \varphi(x) d x\right|=\left|\int_{-\infty}^{\infty} e^{-i \xi(x-i \lambda)} \varphi(x-i \lambda) d x\right| \\
& \leq e^{-\lambda|\xi|} \int_{-\infty}^{\infty}|\varphi(x-i \lambda)| d x=c_{\lambda} e^{-\lambda|\xi|} .
\end{aligned}
$$

To see it when $\xi<0$, replace $\lambda$ by $-\lambda$ in the above estimate.

So $\Phi_{n} \in \mathscr{E}_{\beta}$ for all $n$ and all $\beta>0$. Clearly $\Phi_{n} \rightarrow 1$ uniformly on compact subsets of $(-\infty, \infty)$.

Theorem 5.5. Let $\left(\varphi_{n}\right)$ be an identity sequence, let $\Phi_{n}=\hat{\varphi}_{n}$, and suppose $\beta \geq$ $\alpha>M$.

(i) If $w \in \mathscr{E}_{\alpha}$, then $\varphi_{n} * \check{w}=\left(\Phi_{n} w\right)^{\vee} \in\left(\mathscr{E}_{\beta}\right)^{\vee}(\gamma)$, and $\Phi_{n} w \rightarrow w$ in $\mathscr{E}_{\alpha}$.

(ii) If $u \in L_{1}(\gamma)$, then $\varphi_{n} * u=\left(\Phi_{n} \hat{u}\right)^{\vee} \in\left(\mathscr{E}_{\beta}\right)^{\vee}(\gamma)$ and $\Phi_{n} \hat{u} \rightarrow \hat{u}$ in $\mathscr{E}_{-\beta}$.

Proof. Part (i) is a consequence of the preceding comments, as are the facts that, when $u \in L_{1}(\gamma), \Phi_{n} \hat{u} \in \mathscr{E}_{\beta}$ and $\Phi_{n} \hat{u} \rightarrow \hat{u}$ in $\mathscr{E}_{-\beta}$. Moreover

$$
\begin{aligned}
\varphi_{n} * u(z) & =\int_{\gamma} \varphi_{n}(z-\zeta) u(\zeta) d \zeta \\
& =\int_{\gamma} \frac{1}{2 \pi} \int_{-\infty}^{\infty} \Phi_{n}(\xi) e^{i \xi(z-\zeta)} d \xi u(\zeta) d \zeta \\
& =\frac{1}{2 \pi} \int_{-\infty}^{\infty} \int_{\gamma} e^{-i \xi \zeta} u(\zeta) d \zeta e^{i \xi z} \Phi_{n}(\xi) d \xi \\
& =\frac{1}{2 \pi} \int_{-\infty}^{\infty} \hat{u}(\xi) \Phi_{n}(\xi) e^{i \xi z} d \xi \\
& =\left(\Phi_{n} \hat{u}\right)^{\vee}(z) .
\end{aligned}
$$

We are finally in a position to prove Theorems 5.2 and 5.3.

Proof of Theorem 5.2. (i) Let $u \in L_{1} \cap L_{p}(\gamma)$. Then $\varphi_{n} * u \in\left(\mathscr{E}_{\beta}\right)^{\vee}(\gamma)$ as well as in $L_{1}(\gamma)$ and $L_{p}(\gamma)$, and $\varphi_{n} * u \rightarrow u$ in $L_{1}(\gamma)$ and $L_{p}(\gamma)$. We conclude that $L_{1} \cap L_{p} \cap\left(\mathscr{E}_{\beta}\right)^{\vee}(\gamma)$ is dense in $L_{1} \cap L_{p}(\gamma)$, and therefore in $L_{p}(\gamma)$. So the larger space $L_{p} \cap\left(\mathscr{E}_{\alpha}\right)^{\vee}(\gamma)$ is dense in $L_{p}(\gamma)$. The density of $L_{p} \cap\left(\mathscr{E}_{\beta}\right)^{\vee}(\gamma)$ in $L_{p} \cap\left(\mathscr{E}_{\alpha}\right)^{\vee}(\gamma)$ is also a consequence of the above theorems. The fact that the remaining embeddings are dense was shown previously.

(ii) Reason as above with $L_{p}(\gamma)$ replaced by $C_{0}(\gamma)$.

(iii) Let $u \in L_{1}(\gamma)$ and $w \in \mathscr{E}_{\beta}$. Then $\varphi_{n} * u=\left(\Phi_{n} \hat{u}\right)^{\vee} \in\left(\mathscr{E}_{\beta}\right)^{\vee}(\gamma)$, and therefore, by Theorem 5.1(iii),

$$
\int_{\gamma}\left(\varphi_{n} * u\right)(z) \check{w}(z) d z=\frac{1}{2 \pi} \int_{-\infty}^{\infty}\left(\Phi_{n} \hat{u}\right)(\xi) w(-\xi) d \xi .
$$

Now $\varphi_{n} * u \rightarrow u$ in $L_{1}(\gamma)$ and $\Phi_{n} \hat{u} \rightarrow \hat{u}$ in $\mathscr{E}_{-\beta}$. The result follows.

(iv) This is a consequence of (iii) and the fact that $\left(\mathscr{E}_{\beta}^{2}\right)^{\vee}(\gamma)$ is dense in $L_{1}(\gamma)$.

Proof of Theorem 5.3. Let $u \in L_{p}(\gamma), 1 \leq p \leq \infty$, and $w \in \mathscr{E}_{\beta}$. By Theorem 5.1(iii),

for all $v \in \mathscr{E}_{\beta}^{2}$.

$$
\int_{\gamma} \check{w}(z) \check{v}(z) d z=\frac{1}{2 \pi} \int_{-\infty}^{\infty} w(\xi) v(-\xi) d \xi=\frac{1}{2 \pi}\left\langle w, v_{-}\right\rangle
$$


Suppose that $\check{w}=u \in L_{p}(\gamma)$. Then

$$
\int_{\gamma} \check{w}(z) \check{v}(z) d z=\int_{\gamma} u(z) \check{v}(z) d z=\frac{1}{2 \pi}\left\langle\hat{u}, v_{-}\right\rangle
$$

for all $v \in \mathscr{E}_{\beta}^{2}$ (by the definition of $\hat{u}$ ). So $\hat{u}=w$. Suppose on the other hand that $\hat{u}=w \in \mathscr{E}_{\beta}$. Then

$$
\int_{\gamma} \check{w}(z) \check{v}(z) d z=\frac{1}{2 \pi}\left\langle w, v_{-}\right\rangle=\int_{\gamma} u(z) \check{v}(z) d z
$$

for all $v \in \mathscr{E}_{\beta}^{2}$. In particular,

$$
\int_{\gamma} \varphi_{n}(\zeta-z) \check{w}(z) d z=\int_{\gamma} \varphi_{n}(\zeta-z) u(z) d z
$$

for an identity sequence $\varphi_{n}$. Taking limits for almost all $\zeta \in \gamma$, we conclude that $\check{w}=u$.

We conclude this section by comparing the norms in $L_{p}(\gamma)$ and $L_{p}(\mathbb{R})$ of functions whose Fourier transforms have compact support.

Theorem 5.6. Let $w \in L_{2}(-\infty, \infty)$, and suppose the support of $w$ is contained in the interval $[-S, S]$ for some $S<\infty$. Let $\varepsilon>0$. Then there exists $c_{\varepsilon}>0$ such that, for all $p \in[1, \infty]$,

$$
\|\check{w}\|_{L_{p}(\gamma)} \leq c_{\varepsilon} M^{-1} \exp ((1+\varepsilon) S M)\|\check{w}\|_{L_{p}(\mathbb{R})}
$$

and

$$
\|\check{w}\|_{L_{p}(\mathbb{R})} \leq c_{\varepsilon} M^{-1} \exp ((1+\varepsilon) S M)\|\check{w}\|_{L_{p}(\gamma)} .
$$

Proof. Let $\theta$ denote a $C^{2}$ function on $(-\infty, \infty)$ with support in $[-1-\varepsilon, 1+\varepsilon]$ which equals 1 on a neighbourhood of $[-1,1]$, and $\theta_{S}(\xi)=\theta(\xi / S)$ for $S \geq 1$. Then $\check{\theta}_{S}$ is an entire function which satisfies

$$
\left(1+|\zeta|^{2}\right)\left|\check{\theta}_{S}(\zeta)\right| \leq c_{\varepsilon}\left|\mathcal{I}_{m} \zeta\right|^{-1}\left\{e^{(1+\varepsilon) S\left|\mathcal{I}_{m} \zeta\right|}-1\right\}
$$

for some $c_{\varepsilon}$. So $\left|\check{\theta}_{S}(\zeta)\right| \leq f_{S}(|\zeta|)$ for all $\zeta$ such that $\left|\mathscr{I}_{m} \zeta\right| \leq M$, where $f_{S}$ is an $L_{1}$ function with $\left\|f_{S}\right\|_{1} \leq c_{\varepsilon} M^{-1} \exp ((1+\varepsilon) S M)$.

By adapting the arguments used previously, we find that

$$
\check{w}(z)=\left(\theta_{S} w\right)^{\vee}(z)=\int_{\mathbb{R}} \check{\theta}_{S}(z-\zeta) \check{w}(\zeta) d \zeta
$$

and

$$
\|\check{w}\|_{L_{p}(\gamma)} \leq c_{\varepsilon} M^{-1} \exp ((1+\varepsilon) S M)\|\check{w}\|_{L_{p}(\mathbb{R})} .
$$

The proofs of the identity and the estimate are similar to those in $\S 2$. A corresponding estimate holds if the roles of $\gamma$ and $\mathbb{R}$ are reversed.

Note that the method of proof gives the estimates

$$
\left\|\left(\theta_{S} w\right)^{\vee}\right\|_{L_{p}(\mathbb{R})} \leq c_{p, S}\|\check{w}\|_{L_{p}(\gamma)}, \quad\left\|\left(\theta_{S} w\right)^{\vee}\right\|_{L_{p}(\gamma)} \leq c_{p}, S\|\check{w}\|_{L_{p}(\mathbb{R})}
$$

for every function $w \in \mathscr{E}_{\beta}$.

\section{FOURIER MULTIPLIERS ON CURVES IN A STRIP}

We continue to assume that the curve $\gamma$ is defined by a function $g$ which satisfies $\|g\|_{\infty} \leq M$ and $\left\|g^{\prime}\right\|_{\infty} \leq N$. 
We are now in a position to define Fourier multipliers. Let $1 \leq p \leq \infty$, and choose $\beta<M$.

For $b \in L_{\infty}(-\infty, \infty)$, define

$$
\|b\|_{M_{p}(\gamma)}=\sup \left\{\left\|(b \hat{u})^{\vee}\right\|_{L_{p}(\gamma)} \mid u \in L_{p}(\gamma) \cap\left(\mathscr{E}_{\beta}\right)^{\vee}(\gamma),\|u\|_{p}=1\right\}
$$

and let $M_{p}(\gamma)$ be the space of $L_{\infty}$-functions $b$ for which $\|b\|_{M_{p}(\gamma)}<\infty$. Functions $b \in M_{p}(\gamma)$ are called $L_{p}(\gamma)$-Fourier multipliers.

When $1 \leq p<\infty$ and $b \in M_{p}(\gamma)$, there is a unique bounded linear operator $B$ on $L_{p}(\gamma)$ defined on the dense subspace $L_{p}(\gamma) \cap\left(\mathscr{E}_{\beta}\right)^{\vee}(\gamma)$ by $B u=(b \hat{u})^{\vee}$. When $p=\infty$ and $b \in M_{\infty}(\gamma)$, there is a unique bounded linear operator $B$ on $C_{0}(\gamma)$ defined similarly. If $b_{1}$ and $b_{2}$ are $L_{p}(\gamma)$-Fourier multipliers with corresponding operators $B_{1}$ and $B_{2}$ then $b_{1} b_{2}$ is an $L_{p}(\gamma)$-Fourier multiplier with corresponding operator $B_{1} B_{2}$. The function 1 is in $M_{p}(\gamma)$ with corresponding operator $I$.

The reason for using the space $\mathscr{E}_{\beta}$ in the definition of Fourier multipliers is that if $w \in \mathscr{E}_{\beta}$ and $b \in L_{\infty}(-\infty, \infty)$, then $b w$ is defined and belongs to $\mathscr{E}_{\beta}$. It is a consequence of Theorem 5.2(i) that the definition is independent of the choice of $\beta$ as long as $\beta>M$. A related result is the following.

Proposition. Let $b \in L_{\infty}(-\infty, \infty)$. Then

$$
\|b\|_{M_{p}(\gamma)}=\sup \left\{\left\|(b w)^{\vee}\right\|_{L_{p}(\gamma)} \mid w \in \mathscr{E}_{\beta}^{2},\|\check{w}\|_{L_{p}(\gamma)}=1\right\} .
$$

In particular, $b$ is an $L_{p}(\gamma)$-Fourier multiplier if the right-hand side is finite.

Proof. Suppose that the right-hand side is finite. Let $u \in L_{p}(\gamma) \cap\left(\mathscr{E}_{\beta}\right)^{\vee}(\gamma)$. By Theorem 5.2(i) there is a sequence $w_{n} \in \mathscr{E}_{\beta}^{2}$ such that $\check{w}_{n} \rightarrow u$ in $L_{p}(\gamma) \cap$ $\left(\mathscr{E}_{\beta}\right)^{\vee}(\gamma)$. The sequence $\check{w}_{n}$ is Cauchy in $L_{p}(\gamma)$, so by the hypothesis, $\left(b w_{n}\right)^{\vee}$ is Cauchy in $L_{p}(\gamma)$, which implies that there exists $v \in L_{p}(\gamma)$ such that $\left(b w_{n}\right)^{\vee} \rightarrow v$ in $L_{p}(\gamma)$. Therefore $b w_{n} \rightarrow \hat{v}$ in $\left(\mathscr{E}_{\beta}^{2}\right)^{\prime}$. Also $w_{n} \rightarrow \hat{u}$ in $\mathscr{E}_{\beta}$, so $b w_{n} \rightarrow b \hat{u}$ in $\mathscr{E}_{\beta}$ and hence in $\left(\mathscr{E}_{\beta}^{2}\right)^{\prime}$. We thus see that $\hat{v}=b \hat{u}$ and hence that $\left(b w_{n}\right)^{\vee} \rightarrow(b \hat{u})^{\vee}$ in $L_{p}(\gamma)$. The result now follows.

Proposition. If $1 \leq p \leq \infty$ and $p^{\prime}=\left(1-p^{-1}\right)^{-1}$, then $b \in M_{p}(\gamma)$ if and only if $b_{-} \in M_{p^{\prime}}(\gamma)$, where $b_{-}(\xi)=b(-\xi)$, and $\|b\|_{M_{p}(\gamma)}=\left\|b_{-}\right\|_{M_{p^{\prime}}(\gamma)}$. The corresponding operators $B$ and $B_{-}$are dual in the sense that $\langle B u, v\rangle=\left\langle u, B_{-} v\right\rangle$ for all $u$ and $v$ in the appropriate spaces, and so they have the same spectra: $\sigma(B)=\sigma\left(B_{-}\right)$.

Proof. Apply twice the Parseval's formula in Theorem 5.2(iii).

We also need the following lemma, in which $L_{\text {loc }}(-\infty, \infty)$ denotes the Fréchet space of locally integrable functions on $(-\infty, \infty)$.

Lemma. Suppose $1 \leq p \leq 2$.

(i) If $u \in L_{p}(\gamma)$, then $\hat{u} \in L_{\mathrm{loc}}(-\infty, \infty)$ and the mapping $u \rightarrow \hat{u}$ is continuous from $L_{p}(\gamma)$ to $L_{\mathrm{loc}}(-\infty, \infty)$.

(ii) If $u \in L_{p}(\gamma)$, then $(B u)^{-}=b \hat{u}$.

Proof. Let $\theta_{S}$ be the cut-off function used in Theorem 5.6. By the estimate following that theorem, and by the theorem of Titchmarsh, it follows that

$$
\left\|\theta_{S} w\right\|_{p^{\prime}} \leq c_{p}\left\|\left(\theta_{S} w\right)^{\vee}\right\|_{L_{p}(\mathbb{R})} \leq c_{p, S}\|\breve{w}\|_{L_{p}(\gamma)}
$$


for all $w \in \mathscr{E}_{\beta}$. The density of $L_{p} \cap\left(\mathscr{E}_{\beta}\right)^{\vee}(\gamma)$ in $L_{p}(\gamma)$ can now be used to show that, for every function $u \in L_{p}(\gamma)$, the Fourier transform $\hat{u}$ can be identified with a locally integrable function. Part (ii) then follows.

It is well known that if $b$ is a Lebesgue measurable function on $(-\infty, \infty)$ which satisfies $b \hat{u}=(B u)^{\wedge}$ for some bounded operator $B$ on $L_{p}(\mathbb{R})$ and all $u \in L_{1} \cap L_{p}(\mathbb{R})$, then $b \in L_{\infty}(-\infty, \infty)$ and $\|b\|_{\infty} \leq\|B\|$. We are now in a position to prove a related result for $L_{p}(\gamma)$-Fourier multipliers.

Theorem 6.1. Suppose $1 \leq p \leq \infty$, and let $b \in M_{p}(\gamma)$.

(i) The spectrum $\sigma(B)$ of the operator $B$ corresponding to $b$ satisfies

$$
\sigma(B) \supset \text { ess-range }(b) \text {. }
$$

(ii) $\|b\|_{\infty} \leq\|b\|_{M_{p}(\gamma)}$.

(iii) $M_{p}(\gamma)$ is complete, and hence is a Banach algebra.

Proof. (i) $1 \leq p \leq 2$.

Let $\mathscr{B}(\lambda, \rho)$ and $\mathscr{\mathscr { B }}(\lambda, \rho)$ denote the open and closed balls with centre $\lambda$ and radius $\rho$.

Suppose $\lambda \notin \sigma(B)$. Then there exists $\kappa$ and $\rho>0$ such that $(B-\mu I)$ is invertible and satisfies $\left\|(B-\mu I)^{-1}\right\| \leq \kappa$ for all $\mu \in \overline{\mathscr{B}}(\lambda, \rho)$. We shall use this inequality in a moment to prove that

$$
\left\|\theta_{S} /(b-\mu)\right\|_{\infty} \leq c_{S} \kappa
$$

where $\theta_{S}$ is the cut-off function used in Theorem 5.6 (with $\varepsilon<1$ ) and $c_{S}$ is a constant which depends on $S$ (and $M$ and $p$ ) but not on $\mu$. Thus $\{b(\xi) \mid-S \leq \xi \leq S\} \cap \mathscr{B}\left(\mu,\left(c_{S} \kappa\right)^{-1}\right)$ has measure zero. On covering $\mathscr{\mathscr { B }}(\lambda, \rho)$ with finitely many balls of the form $\mathscr{B}\left(\mu\left(c_{S} \kappa\right)^{-1}\right)$, we see that $\{b(\xi) \mid-S \leq$ $\xi \leq S\} \cap \mathscr{B}(\lambda, \rho)$ has measure zero. On considering a sequence of numbers $S$ which tends to infinity, we find that

$$
\mathscr{B}(\lambda, \rho) \cap \text { ess-range }(b)=\varnothing .
$$

Therefore ess-range $(b) \subset \sigma(B)$.

It remains for us to prove $(*)$. We do this by defining a bounded operator $F_{S, \mu}$ on $L_{p}(\mathbb{R})$ which satisfies

$$
(b-\mu)\left(F_{S, \mu} u\right)^{\wedge}=\theta_{S} \hat{u}, \quad u \in L_{p}(\mathbb{R}),
$$

and $\left\|F_{S, \mu}\right\| \leq c_{S} \kappa$ where $c_{S}$ depends on $S$ but not on $\mu$, and then using the comment preceding the theorem to deduce $(*)$.

Define $F_{S, \mu}$ by $F_{S, \mu} u=\check{\theta}_{2 S} *(B-\mu I)^{-1}\left(\check{\theta}_{S} * u\right)$ for $u \in L_{p}(\mathbb{R})$. Then by the final estimates in $\S 5$,

$$
\begin{aligned}
\left\|F_{S, \mu} u\right\|_{L_{p}(\mathbf{R})} & \leq c_{p, 2 S}\left\|(B-\mu I)^{-1}\left(\check{\theta}_{S} * u\right)\right\|_{L_{p}(\gamma)} \\
& \leq \kappa c_{p, 2 S}\left\|\check{\theta}_{S} * u\right\|_{L_{p}(\gamma)} \leq \kappa c_{p, S} c_{p, 2 S}\|u\|_{L_{p}(\mathbf{R})}
\end{aligned}
$$

as required. We leave it to the reader to check $(* *)$.

This completes the proof of (i) when $1 \leq p \leq 2$. The result for $2<p \leq \infty$ follows by duality on applying the preceding proposition.

$$
\begin{aligned}
\|b\|_{\infty} & =\sup \{|\lambda| \mid \lambda \in \text { ess-range }(b)\} \\
& \leq \sup \{|\lambda| \mid \lambda \in \sigma(B)\} \leq\|B\|=\|b\|_{M_{p}(\gamma)} .
\end{aligned}
$$


(iii) What remains to be shown is that $M_{p}(\gamma)$ is complete. Let $\left(b_{n}\right)$ be a Cauchy sequence in $M_{p}(\gamma)$, and let $B_{n}$ be the operator on $L_{p}(\gamma)$ corresponding to $b_{n}$. Then $B_{n}$ converges in the operator norm to an operator $B$ on $L_{p}(\gamma)$, and, by (ii), $b_{n}$ converges in the $L_{\infty}$ norm to a function $b \in L_{\infty}(-\infty, \infty)$. It is straightforward to show that $b \in M_{p}(\gamma), b_{n} \rightarrow b$ in $M_{p}(\gamma)$, and $B$ is the operator corresponding to $b$.

\section{EXAMPLES OF $L_{p}(\gamma)$-FOURIER MULTIPLIERS}

We continue to assume that the curve $\gamma$ is defined by a function $g$ which satisfies $\|g\|_{\infty} \leq M$ and $\left\|g^{\prime}\right\|_{\infty} \leq N$.

First consider convolutions.

Theorem 7.1. Suppose $1 \leq p \leq \infty$. Let $\varphi$ be a holomorphic function defined on a simply connected open neighbourhood of $\Gamma=\gamma-\gamma$ which satisfies $|\varphi(z)| \leq$ $\psi(|\mathscr{R e} z|)$ where $\int_{0}^{\infty} \psi(s) d s<\infty$. Define

$$
b(\xi)=\int_{\mathbb{R}} e^{-i x \xi} \varphi(x) d x, \quad-\infty<\xi<\infty .
$$

Then $b \in M_{p}(\gamma)$ and the corresponding operator $B$ is given by

$$
B u(z)=\int_{\gamma} \varphi(z-\zeta) u(\zeta) d \zeta \quad \text { a.e. }
$$

for all $u \in L_{p}(\gamma)$ (if $\left.1 \leq p<\infty\right)$ or $u \in C_{0}(\gamma)$ (if $\left.p=\infty\right)$. Moreover

$$
\begin{aligned}
\|b\|_{M_{p}(\gamma)} & \leq \sup _{z \in \gamma}\left\{\int_{\gamma}|\varphi(z-\zeta)||d \zeta|\right\}^{1 / p^{\prime}} \sup _{\zeta \in \gamma}\left\{\int_{\gamma}|\varphi(z-\zeta) \| d z|\right\}^{1 / p} \\
& \leq 2 \sqrt{1+N^{2}} \int_{0}^{\infty} \psi(s) d s .
\end{aligned}
$$

Proof. It can easily be verified that $\varphi * \check{w}=(b w)^{\vee}$ for all $w \in \mathscr{E}_{\beta}^{2}$. On making use of Theorems 2.1 and 5.2, the result follows.

We could have been a little more ambitious in the preceding section, and treated multiplier spaces $M_{p}\left(\gamma_{1}, \gamma_{2}\right)$ where $\gamma_{1}$ and $\gamma_{2}$ are two curves of the type under consideration. Let us do so briefly now.

$$
M_{p}\left(\gamma_{1}, \gamma_{2}\right)=\left\{b \in L_{\infty}(-\infty, \infty) \mid\|b\|_{M_{p}\left(\gamma_{1}, \gamma_{2}\right)}<\infty\right\}
$$

where

$$
\|b\|_{M_{p}\left(\gamma_{1}, \gamma_{2}\right)}=\sup \left\{\left\|(b w)^{\vee}\right\|_{L_{p}\left(\gamma_{2}\right)} /\|\check{w}\|_{L_{p}\left(\gamma_{1}\right)} \mid w \in \mathscr{E}_{\beta}, \check{w} \in L_{p}\left(\gamma_{1}\right)\right\} .
$$

If $\gamma_{3}$ is a third such curve, and $b \in M_{p}\left(\gamma_{1}, \gamma_{2}\right), b_{1} \in M_{p}\left(\gamma_{2}, \gamma_{3}\right)$, then $b_{1} b \in$ $M_{p}\left(\gamma_{1}, \gamma_{3}\right)$ and

$$
\left\|b_{1} b\right\|_{M_{p}\left(\gamma_{1}, \gamma_{3}\right)} \leq\left\|b_{1}\right\|_{M_{p}\left(\gamma_{2}, \gamma_{3}\right)}\|b\|_{M_{p}\left(\gamma_{1}, \gamma_{2}\right)} .
$$

Theorem 7.1 can be adapted to this setting provided $\varphi$ is defined on a neighbourhood of $\gamma_{2}-\gamma_{1}$. We give an example. Let $\varphi_{\beta}(z)=\beta \pi^{-1}\left(z^{2}+\beta^{2}\right)^{-1}$. Then $b_{\beta}(\xi)=\exp (-\beta|\xi|)$. If $\beta>M$, then $b_{\beta} \in M_{p}(\gamma, \mathbb{R})$ and

$$
\begin{aligned}
\left\|b_{\beta}\right\|_{M_{p}(\gamma, \mathbb{R})} & \leq \sup _{z \in \gamma}\left\{\int_{\mathbb{R}}\left|\varphi_{\beta}(z-x)\right| d x\right\}^{1 / p^{\prime}} \sup _{x \in \mathbb{R}}\left\{\int_{\gamma}\left|\varphi_{\beta}(z-x)\right||d z|\right\}^{1 / p} \\
& \leq \beta\left(\beta^{2}-M^{2}\right)^{-1}\left(1+N^{2}\right)^{1 / 2 p} .
\end{aligned}
$$


Also, $b_{\beta} \in M_{p}(\mathbb{R}, \gamma)$ and

$$
\left\|b_{\beta}\right\|_{M_{p}(\mathbb{R}, \gamma)} \leq \beta\left(\beta^{2}-M^{2}\right)^{-1}\left(1+N^{2}\right)^{1 / 2 p^{\prime}} .
$$

It is a consequence of Theorem 5.7 that every $L_{p}(\mathbb{R})$-Fourier multiplier with compact support is an $L_{p}(\gamma)$-Fourier multiplier. Somewhat more general is the following theorem.

Theorem 7.2. Suppose $1 \leq p \leq \infty$. For $b \in L_{\infty}(\mathbb{R})$, define

$$
f_{\beta}(\xi)=b(\xi) \exp (2 \beta|\xi|) \text {. }
$$

If $f_{\beta} \in M_{p}(\mathbb{R})$ for some $\beta>M$, then $b \in M_{p}(\gamma)$ and

$$
\|b\|_{M_{p}(\gamma)} \leq \beta^{2}\left(\beta^{2}-M^{2}\right)^{-2}\left(1+N^{2}\right)^{1 / 2}\left\|f_{\beta}\right\|_{M_{p}(\mathbb{R})} .
$$

Proof. Write $b=b_{\beta} f_{\beta} b_{\beta}$ with $b_{\beta}$ defined above. Then, from above,

$$
\|b\|_{M_{p}(\gamma)} \leq\left\|b_{\beta}\right\|_{M_{p}(\mathbb{R}, \gamma)}\left\|f_{\beta}\right\|_{M_{p}(\mathbb{R})}\left\|b_{\beta}\right\|_{M_{p}(\gamma, \mathbb{R})} .
$$

The following results are immediate consequences of this result and of known results about $L_{p}(\mathbb{R})$-Fourier multipliers.

(1) $1<p<\infty$. The characteristic function $\chi_{J}$ of every bounded interval $J=[a, b]$ is an $L_{p}(\gamma)$-Fourier multiplier. The corresponding operator $B$ is given by

$$
B u(z)=\frac{i}{\pi} \int_{\gamma}\left\{e^{i(z-\zeta) a}-e^{i(z-\zeta) b}\right\} \frac{1}{z-\zeta} u(\zeta) d \zeta
$$

for all $u \in L_{p}(\gamma)$.

(2) $p=2$. Every Lebesgue measurable function $b$ which satisfies $|b(\xi)| \leq$ $c \exp (-2 \beta|\xi|)$ for some $\beta>M$ and $c \geq 0$ is a $L_{2}(\gamma)$-Fourier multiplier.

(3) $1<p<\infty$. If $|b(\xi)| \leq c \exp (-2 \beta|\xi|)$ for some $\beta>M$ and $c \geq 0$, and, for all $a>0$.

$$
\int_{a}^{2 a}|d g(x)| \leq \text { const. }
$$

where $g(\xi)=b(\xi) \exp (2 \beta|\xi|)$, then $b \in M_{p}(\gamma)$.

The third result is our analogue of the Marcinkiewicz multiplier theorem. Clearly, more such results could be listed.

Our next task is to investigate the connection between multipliers and functions of $D_{\gamma}$. We use the notation and definitions of $\S \S 3$ and 4. Theorem 7.4 connects our results on $b\left(D_{\gamma}\right)$ with the results of Coifman and Meyer in [2]. Also see [6].

Theorem 7.3. Suppose $1 \leq p \leq \infty$. If a function $b \in L_{\infty}(\mathbb{R})$ can be extended to a function $b \in \Psi\left(S_{\mu}^{0}\right)$ for some $\mu>\tan ^{-1} N$, then $b \in M_{p}(\gamma)$ and the corresponding operator $B$ on $L_{p}(\gamma), 1 \leq p<\infty$, or on $C_{0}(\gamma)$ satisfies $B=$ $b\left(D_{\gamma}\right)$.

Proof. This is an immediate consequence of the preceding theorem and the results stated in $\S 3$.

Theorem 7.4. Suppose $1<p<\infty$. If a function $b \in L_{\infty}(\mathbb{R})$ can be extended to a function $b \in H_{\infty}\left(S_{\mu}^{0}\right)$ for some $\mu>\tan ^{-1} N$, then $b \in M_{p}(\gamma)$ and the corresponding operator $B$ on $L_{p}(\gamma), 1<p<\infty$, satisfies $B=b\left(D_{\gamma}\right)$. 
Proof. Let $b \in H_{\infty}\left(S_{\mu}^{0}\right)$. We need to prove that $(b w)^{\vee}=b\left(D_{\gamma}\right) \check{w}$ for all $w \in \mathscr{E}_{\beta}^{2}$. So fix $w \in \mathscr{E}_{\beta}^{2}$. Choose a sequence $\left(b_{n}\right)$ of functions in $\Psi\left(S_{\mu}^{0}\right)$ which satisfies

(i) $\left\|b_{n}\right\|_{\infty} \leq$ const. $n=1,2,3, \ldots$;

(ii) for all $0<\delta<\Delta<\infty, b_{n} \rightarrow b$ uniformly on $\left\{\zeta \in S_{\mu}^{0}|\delta \leq| \zeta \mid \leq \Delta\right\}$. As stated in $\S 4, b_{n}\left(D_{\gamma}\right) \check{w} \rightarrow b\left(D_{\gamma}\right) \check{w}$ in $L_{p}(\gamma)$. Moreover, it is readily seen that $b_{n} w \rightarrow b w$ in $\mathscr{E}_{\beta}^{2}$, so $\left(b_{n} w\right)^{\vee} \rightarrow(b w)^{\vee}$ in $L_{p}(\gamma)$. By Theorem 7.3, $b_{n}\left(D_{\gamma}\right) \check{w}=\left(b_{n} w\right)^{\vee}$. We conclude that $b\left(D_{\gamma}\right) \check{w}=(b w)^{\vee}$ as required.

An explicit formula for $b\left(D_{\gamma}\right)$ was given in $\S 4$. See [6] for details.

Corollary. Suppose $1<p<\infty$. The characteristic function $\chi_{J}$ of every interval $J$ is an $J_{p}(\gamma)$-Fourier multiplier.

Proof. We have already seen this in the case when $J$ is bounded. On the other hand, when $J=(0, \infty)$ or $(-\infty, 0)$, the boundedness of $\chi_{J}$ is an immediate consequence of Theorem 7.4. The case when $J$ is an arbitrary unbounded interval can easily be reduced to a combination of these two situations.

The bounded operators $E_{J}$ corresponding to $\chi_{J}$ are spectral projections of $D_{\gamma}$. Note however that they have not been shown to be uniformly bounded, and indeed it can be proved that $\left\|E_{(-\infty, a]}\right\| \geq c_{\varepsilon} \exp \left(|a|\left(\|g\|_{\infty}-\varepsilon\right)\right)$. So, by the uniform boundedness principle, $E_{(-\infty, a]}$ does not converge strongly to the identity as $a \rightarrow \infty$. Nevertheless they do tie in with the general spectral theories developed by Ricker and Kluvánek.

We conclude with the remark that the classical version of Theorem 7.4 (with $\gamma=\mathbb{R}$ ) is an immediate consequence of the Marcinkiewicz multiplier theorem, whereas there is quite a disparity between Theorem 7.4 for general $\gamma$ and the result (3) following Theorem 7.2. It may be of interest to look for conditions on $b$ to be in $M_{p}(\gamma)$ which tie together both types of results.

\section{APPENDIX}

In the approximation arguments of $\S 5$ we used the following result. The proof below is a modification of one given by Michael Benedicks and Peter Jones.

Theorem. Let $0<\mu<\pi / 2$. There exists an entire holomorphic function $\varphi$ which satisfies, for some constant $c_{\mu}$,

$$
\int_{-\infty}^{\infty} \varphi(x) d x=1
$$

and

$$
|\varphi(z)| \leq \frac{c_{\mu}}{1+x^{2}}, \quad z=x+i y \in S_{\mu}^{0} .
$$

Proof. First let $f$ be the holomorphic function defined on the upper-half plane by $f(z)=(i+z)^{-2} \exp \left((-i z)^{\lambda}\right)$ where $\lambda$ satisfies $\pi / 2 \lambda<\pi / 2-\mu$. Note that

(i) $|f(z)|=|i+z|^{-2}$ for all $z \in \delta$, where $\delta$ is the curve given by $\left|\frac{1}{2} \pi-\arg z\right|=\pi / 2 \lambda$, and

(ii) $|f(i y)| \rightarrow \infty$ as $y \rightarrow+\infty$. (See Figure 2.)

Next define $G$ by

$$
G(z)= \begin{cases}\frac{1}{2 \pi i} \int_{\delta} \frac{1}{z-\zeta} f(\zeta) d \zeta, & z \text { below } \delta \\ \frac{1}{2 \pi i} \int_{\delta} \frac{1}{z-\zeta} f(\zeta) d \zeta+f(z), & z \text { above } \delta\end{cases}
$$




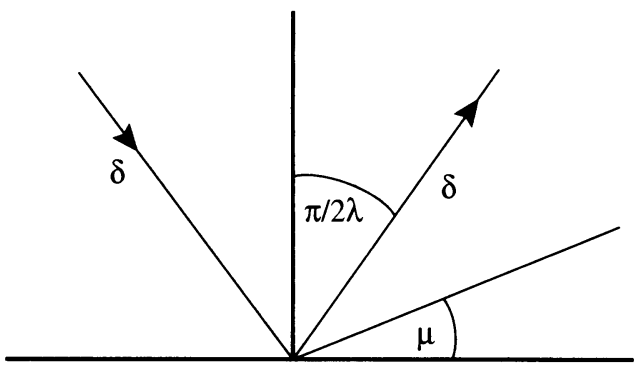

0

Figure 2

The function $G$ extends to a continuous function on $\mathbb{C}$ which is in fact an entire holomorphic function. It is bounded below $\delta$ and unbounded above $\delta$ (and hence not identically zero).

Finally, define $\varphi$ by $\varphi(z)=\kappa G^{\prime}(z) \overline{G^{\prime}(\bar{z})}$ where $\kappa$ is a normalizing factor. Then $\varphi$ has the required properties.

Added in proof. A discussion of Fourier multipliers on $L_{p}(\Sigma)$, where $\Sigma$ is a Lipschitz surface of the form $\Sigma=\left\{(\mathbf{s}, g(\mathbf{s})) \mid s \in \mathbb{R}^{m}\right\}$ with $\|\nabla g\|_{\infty} \leq N$, is contained in Clifford algebras, Fourier transforms, and singular convolution operators on Lipschitz surfaces by Chun Li, Alan McIntosh and Tao Qian, Macquarie Mathematics Report No. 91-087.

\section{REFERENCES}

1. R. R. Coifman, A. McIntosh and Y. Meyer, L'intégral de Cauchy définit un opérateur borné sur $L^{2}$ pour les courbes Lipschitziennes, Ann. of Math. (2) 116 (1982), 361-387.

2. R. R. Coifman and Y. Meyer, Fourier analysis of multilinear convolutions, Calderón's theorem, and analysis on Lipschitz curves, Lecture Notes in Math., vol. 779, Springer-Verlag, Berlin, 1980, pp. 104-122.

3. M. Cowling, I. Doust, A. McIntosh and A. Yagi, Banach space operators with an $H_{\infty}$ functional calculus (in preparation).

4. Alan McIntosh, Operators which have an $H_{\infty}$-functional calculus, Miniconference on Operator Theory and Partial Differential Equations, (Canberra, 1986), Proc. Centre Math. Anal. Austral. Nat. Univ., 14, ANU, Canberra, 1986, pp. 210-231.

5. Alan McIntosh and Tao Qian, Fourier theory on Lipschitz curves, Miniconference on Harmonic Analysis, (Canberra, 1987), Proc. Centre Math. Anal., 15, ANU, Canberra, 1987, pp. 157-166.

6. __ Convolution singular integral operators on Lipschitz curves, Lecture Notes in Math., vol. 1494, Springer-Verlag, Berlin, 1991, pp. 142-162.

7. Elias M. Stein, Singular integrals and differentiability properties of functions, Princeton Univ. Press, Princeton, N.J., 1970.

School of Mathematics, Physics, Computing and Electronics, Macquarie University, N.S.W. 2109, Australia

E-mail address: alan@macadam.mpce.mq.edu.au

School of Mathematical Sciences, Flinders University, Bedford Park, S.A. 5042, AusTRALIA

E-mail address: t.qian@cc.flinders.edu.au 